\author{
Ecología
}

\title{
Tricópteros asociados a siete afluentes de la Sierra Nevada de Santa Marta, Colombia
}

\author{
Caddisflies associated to seven streams of the Sierra Nevada de Santa Marta, Colombia \\ Ana Milena Jaimes-Contreras * y Cristian Granados-Martínez \\ Fundación para la Investigación en Biodiversidad del Caribe Colombiano, RIWISAKA-Grupo de Investigación en Ecología Neotropical (GIEN), Universidad \\ del Magdalena, Carrera 32 n. ${ }^{\circ}$ 22. 08 Apartado postal 2-1-21630, 470004, Santa Marta, Colombia \\ Recibido el 29 de julio de 2015; aceptado el 18 de noviembre de 2015 \\ Disponible en Internet el 17 de abril de 2016
}

\begin{abstract}
Resumen
En la actualidad, la intensidad de las actividades agrícolas está aumentando en zonas cercanas a las fuentes hídricas por la disminución de las lluvias atribuidas al aumento en el fenómeno de cambio climático. El objetivo del presente trabajo fue analizar la estructura de las larvas del orden Trichoptera en 7 afluentes en la Sierra Nevada de Santa Marta y su relación con variables fisicoquímicas. Las muestras fueron recolectadas usando una red Surber con ojo de malla de $250 \mu \mathrm{m}$, se recolectaron al azar tomando 5 réplicas para un total de $0.9 \mathrm{~m}^{2}$ de área muestreada para cada río. Se encontró un total de 868 individuos del orden Trichoptera, distribuidos en 9 familias y 15 géneros. Hydropsychidae y Leptoceridae fueron las más representativas en todo el muestreo, con una abundancia del 66 y 14\%, respectivamente. En términos generales, las larvas mostraron variación a nivel espacial. A pesar de los valores ligeramente altos de conductividad y de nutrientes para algunos afluentes, los valores de diversidad y abundancia reflejan el buen estado de los afluentes muestreados en la Sierra Nevada de Santa Marta. Los resultados de este trabajo indican que los afluentes estudiados constituyen importantes reservorios de diversidad de tricópteros.

Derechos Reservados (C) 2016 Universidad Nacional Autónoma de México, Instituto de Biología. Este es un artículo de acceso abierto distribuido bajo los términos de la Licencia Creative Commons CC BY-NC-ND 4.0.
\end{abstract}

Palabras clave: Variables fisicoquímicas; Afluentes; Diversidad; Abundancia

\begin{abstract}
Nowadays, the intensity of agricultural activities is increasing in areas near water sources due to decreasing rains attributed to the increase in the phenomenon of climate change. The aim of this study was to analyze the structure of the order Trichoptera larvae in 7 tributaries in the Sierra Nevada de Santa Marta and their relationship with physicochemical variables. The samples were collected using a Surber's net with a 250 microns mesh, collected randomly taking 5 replicates for a total of $0.9 \mathrm{~m}^{2}$ for each river. A total of 868 individuals of the order Trichoptera, distributed in 9 families and 15 genera was found. Hydropsychidae and Leptoceridae were the best represented in the sampling, with 66 and $14 \%$, respectively. Overall, the larvae showed a spatial variation. Despite the slightly high values of conductivity and nutrients in some tributaries, the values of diversity and abundance of caddisflies reflect the good state of tributaries in the Sierra Nevada de Santa Marta. The results of this study indicate that the tributaries are important reservoirs of caddisfly diversity.

All Rights Reserved (C 2016 Universidad Nacional Autónoma de México, Instituto de Biología. This is an open access item distributed under the Creative Commons CC License BY-NC-ND 4.0.
\end{abstract}

Keywords: Physicochemical variables; Tributaries; Diversity; Abundance

* Autora para correspondencia.

Correo electrónico: amilenajaimes@gmail.com (A.M. Jaimes-Contreras).

La revisión por pares es responsabilidad de la Universidad Nacional Autónoma de México.

\section{Introducción}

Los tricópteros son organismos con un ciclo de vida donde las formas inmaduras son acuáticas y los adultos son voladores; son holometábolos, ya que desarrollan estados de huevo, larva, pupa y adulto (Posada-García y Roldán-Pérez, 2003). Las larvas están 
presentes en diferentes hábitats, principalmente en ecosistemas lóticos, presentan generalmente 5 estadios larvales con tamaños entre 1 y $30 \mathrm{~mm}$, y pueden ser libres o vivir en refugios que construyen con seda y diferentes materiales orgánicos e inorgánicos (López-Delgado, Vásquez-Ramos y Reinoso-Flórez, 2015). Los tricópteros desempeñan un papel muy importante en los ecosistemas acuáticos, ya que forman parte de la cadena trófica y son el principal alimento de una gran variedad de peces, aves y otros vertebrados que se encuentran cerca de los cuerpos de agua (Domínguez y Fernández, 2009; Wiggins, 1996a). Además, se consideran como potencialmente útiles en estudios sobre calidad del agua debido a su sensibilidad frente a los cambios físicos y químicos (Guevara, López, Reinoso y Villa-Navarro, 2006; Valero, Durant y Arellano, 2001).

La estructura de las comunidades de insectos acuáticos se ha estudiado de forma extensiva en las zonas templadas (Allan y Castillo, 2007). En la región Neotropical, en particular en América del Sur, en la última década se desarrollaron trabajos taxonómicos y ecológicos que han aportado al conocimiento de los tricópteros (Bispo, Oliveira, Crisci-Bispo y Sousa, 2004; Diniz-Filho, Oliveira y Silva, 1998; Domínguez y Fernández, 2009; Fernández y Domínguez, 2001; Huamantinco y Nessimian, 1999; Muñoz-Quesada, 2004; Oliveira y Froehlich, 1996; Oliveira, Bispo, Crisci y Sousa, 1999; Spies, Froehlich y Kotzian, 2006), sin embargo, la información es escasa. En Colombia, los estudios de tricópteros se han desarrollado de forma importante en la región Andina (Guevara, 2004; Guevara, Reinoso y Villa-Navarro, 2006; López y Reinoso, 2008; MuñozQuesada, 2004; Posada-García y Roldán-Pérez, 2003; Quintero y Rojas, 1987; Ramírez, Vásquez, Reinoso y Guevara, 2007, 2008a,b; Reinoso, 2001; Vásquez y Ramírez, 2008; Vásquez, Ramírez, Reinoso y Guevara, 2006, 2007, 2008a,b), mientras que en la región Caribe y en particular en la Sierra Nevada de Santa Marta (SNSM) los estudios han sido insuficientes (Serna, Tamaris-Turizo y Gutiérrez-Moreno, 2015). Actualmente, la SNSM presenta un alto grado de impacto ambiental en sus cuencas, debido a varios factores de origen antrópico como la deforestación, el uso de tierras de cultivo, el cambio climático y la contaminación (Manjarrés-García y Manjarrés-Pinzón, 2004; Viloria de la Hoz, 2006). Nuestro objetivo fue analizar la estructura de las larvas del orden Trichoptera en 7 afluentes en la SNSM y su relación con variables fisicoquímicas.

\section{Materiales y métodos}

El estudio se realizó en 7 afluentes pertenecientes a los flancos norte y oeste de la SNSM (fig. 1): río Piedras, río Mendihuaca, quebrada Cherua, río Fundación, quebrada El

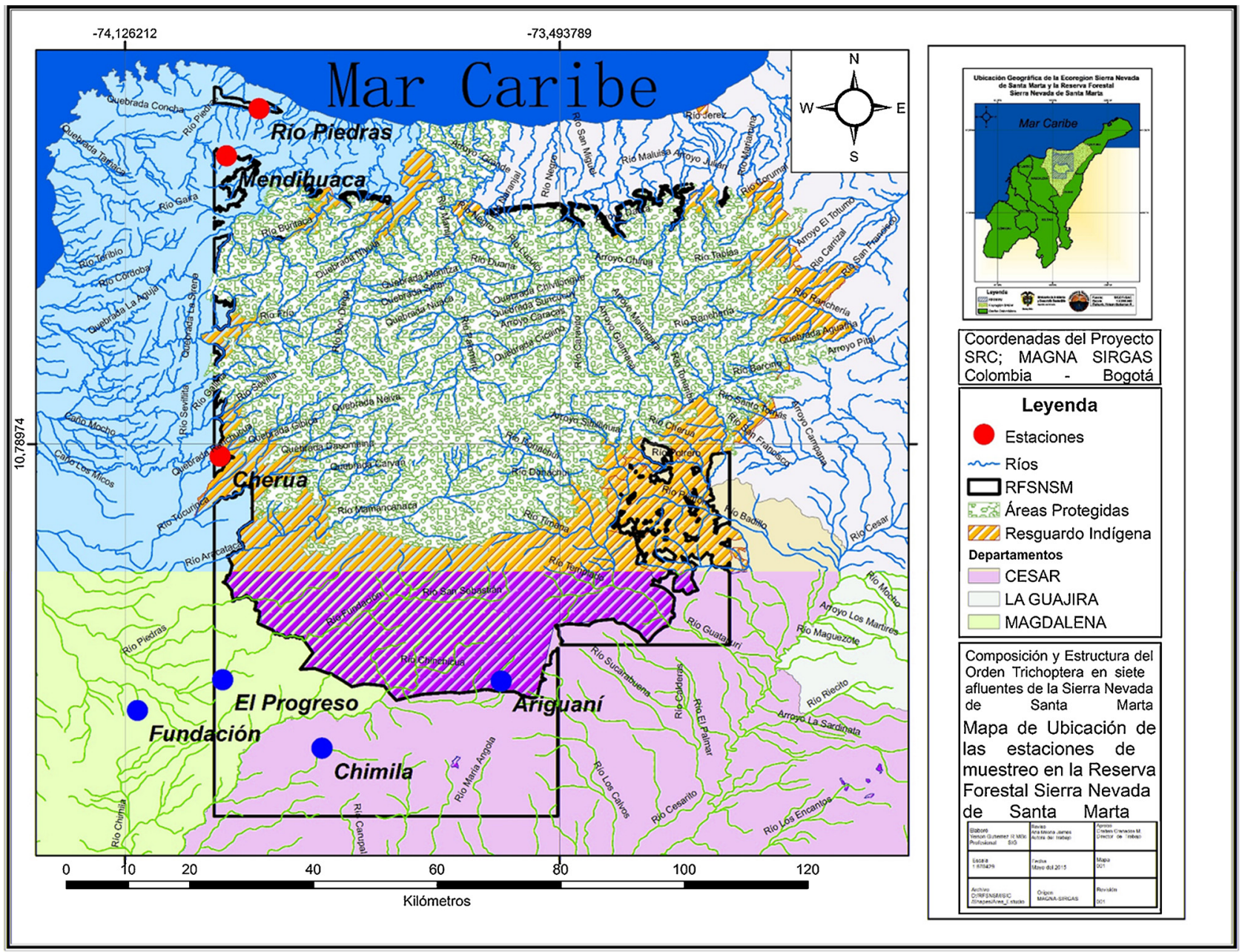

Figura 1. Mapa del área de estudio. 
Tabla 1

Coordenadas de las estaciones de muestreo.

\begin{tabular}{lll}
\hline Estación & Coordenadas & Altitud (m snm) \\
\hline Piedras & $11^{\circ} 16^{\prime} 66.5^{\prime \prime} \mathrm{N}, 73^{\circ} 55^{\prime} 97.0^{\prime \prime} \mathrm{O}$ & 51 \\
Mendihuaca & $11^{\circ} 12^{\prime} 36.6^{\prime \prime} \mathrm{N}, 73^{\circ} 58^{\prime} 41.2^{\prime \prime} \mathrm{O}$ & 865 \\
Cherua & $10^{\circ} 46^{\prime} 25.9^{\prime \prime} \mathrm{N}, 76^{\circ} 58^{\prime} 59.7^{\prime \prime} \mathrm{O}$ & 1,363 \\
Fundación & $10^{\circ} 26^{\prime} 42.0^{\prime \prime} \mathrm{N}, 73^{\circ} 59^{\prime} 19.0^{\prime \prime} \mathrm{O}$ & 283 \\
El Progreso & $10^{\circ} 28^{\prime} 98.3^{\prime \prime} \mathrm{N}, 73^{\circ} 54^{\prime} 77.6^{\prime \prime} \mathrm{O}$ & 872 \\
Chimila & $10^{\circ} 20^{\prime} 37.7^{\prime \prime} \mathrm{N}, 73^{\circ} 49^{\prime} 23.77^{\prime} \mathrm{O}$ & 441 \\
Ariguaní & $10^{\circ} 26^{\prime} 41.3^{\prime \prime} \mathrm{N}, 73^{\circ} 34^{\prime} 44.4^{\prime \prime} \mathrm{O}$ & 1,205 \\
\hline
\end{tabular}

Progreso, quebrada Chimila y río Ariguaní (tabla 1). Cada uno de los afluentes posee un grado de intervención antrópica, debido al uso de tierras para cultivo, presencia de caminos construidos o zonas de captación o descarga de agua. La estación de Mendihuaca constituye una excepción, con un bosque de ribera en buen grado de conservación. Igualmente, cada río posee un determinado tipo de cobertura en el lecho; río Piedras: lecho con cobertura de arenas, cantos rodados y rocas; río Mendihuaca: lecho con cobertura de cantos rodados, rocas y gravas, con dominancia del primer elemento; quebrada Cherua: lecho con cobertura de cantos rodados, arenas y rocas; río Fundación: lecho con cobertura principalmente rocosa; quebrada El Progreso: lecho con cobertura de cantos rodados, arenas y rocas; quebrada Chimila: lecho con cobertura principalmente rocosa, con menor proporción de cantos rodados y arena; y río Ariguaní: lecho con cobertura de canto rodado, arenas y rocas.

En cada estación se registraron distintas variables ambientales. Con ayuda de una sonda multiparámetros (WTW 350) se midieron las siguientes variables: temperatura del agua $\left({ }^{\circ} \mathrm{C}\right)$, oxígeno disuelto (OD mg/l), conductividad $(\mu \mathrm{S} / \mathrm{cm}), \mathrm{pH}$ y sólidos disueltos (ppm). Por otra parte, en cada estación se tomaron muestras de agua de $500 \mathrm{ml}$ en botellas plásticas, las cuales fueron refrigeradas para su posterior análisis en los laboratorios de la Universidad del Magdalena, donde se analizaron nutrientes y componentes - nitritos, nitratos y fosfatos-.

El muestreo biológico se realizó entre octubre y diciembre del 2013, correspondiente al periodo de lluvias en la región. Se seleccionaron 3 tramos de $100 \mathrm{~m}$ en cada uno de los afluentes, teniendo en cuenta a su vez el criterio de heterogeneidad microgeomorfológica y el sistema de mansos y remansos (Dunne y Leopold, 1978; Frissell, Liss, Warren y Hurley, 1986). Posteriormente, se hizo una selección de rápidos y remansos de acuerdo con Frissell et al. (1986). Los macroinvertebrados bentónicos fueron recolectados usando una red Surber con ojo de malla de $250 \mu \mathrm{m}$, las muestras de la red fueron recolectadas al azar tomando 5 réplicas para un total de $0.9 \mathrm{~m}^{2}$ de área muestreada para cada río. El material retenido fue guardado en bolsas de polietileno debidamente rotuladas y fijadas con etanol al $96 \%$. La limpieza y separación del material se hizo en el laboratorio con ayuda de bandejas plásticas. La identificación se hizo utilizando un estereoscopio de marca Nikon SMZ-645 y claves taxonómicas especializadas (Fernández y Domínguez, 2001; Springer, Ramírez y Hanson, 2010; Wiggins, 1996b).

Se realizaron análisis de datos exploratorios que incluyeron tablas dinámicas y gráficos de barra. Se analizó la estructura de las comunidades de macroinvertebrados mediante índices de diversidad conocidos: dominancia $(\mathrm{D}=)$, Shanon-Weaver $(\mathrm{H}=)$ y equidad de Pielou $(\mathrm{J}=)$ (Ramírez y Viña, 1998). Para determinar la relación entre las comunidades de tricópteros y las variables fisicoquímicas (temperatura del agua, OD, conductividad, $\mathrm{pH}$ y sólidos disueltos), se realizó un análisis de correspondencia canónica utilizando PAST versión 2.3 (Hammer, Harper y Ryan, 2001).

\section{Resultados}

Los valores registrados de las variables fisicoquímicas y las concentraciones de nutrientes se observan en la tabla 2. En términos generales en todas las estaciones el $\mathrm{pH}$ fue ligeramente básico, los menores valores se encontraron en el Progreso y Ariguaní (7.6), mientras que los mayores se hallaron en las estaciones de río Piedras y Cherua con 8.6 y 8.2. La menor temperatura se registró en la estación Cherua con $19.3^{\circ} \mathrm{C}$ y la mayor en la estación de río Piedras con $30^{\circ} \mathrm{C}$. Los valores de conductividad fueron heterogéneos, el menor se registró en la estación Chimila con $73.5 \mu \mathrm{S} / \mathrm{cm}$, mientras que el mayor lo registró la estación de río Ariguaní con $173.5 \mu \mathrm{S} / \mathrm{cm}$. Los valores de OD en general fueron altos; el menor valor de esta variable se presentó en la estación El Progreso con $6.2 \mathrm{mg} / \mathrm{l}$, mientras que el mayor fue hallado en Ariguaní con $7.7 \mathrm{mg} / \mathrm{l}$. El menor valor de sólidos disueltos se presentó en la estación Mendihuaca con 38.5 ppm, mientras que el mayor fue en Chimila con $130 \mathrm{ppm}$. Respecto a los nutrientes, los valores de nitrito variaron entre 0.1 y $9.7 \mathrm{mg} / \mathrm{l}$, el mayor valor lo registró el río Fundación y el menor la estación Ariguaní. Para los valores de nitratos, estos variaron de 0.5 a $4.4 \mathrm{mg} / \mathrm{l}$, donde el menor valor fue registrado en Ariguaní y el mayor en río Piedras. Los fosfatos variaron de 1 a $3.9 \mathrm{mg} / \mathrm{l}$, donde el menor valor de fosfatos se registró en Ariguaní y el mayor en río Piedras (tabla 2).

Tabla 2

Valores de las variables fisicoquímicas y de nutrientes de las estaciones de muestreo.

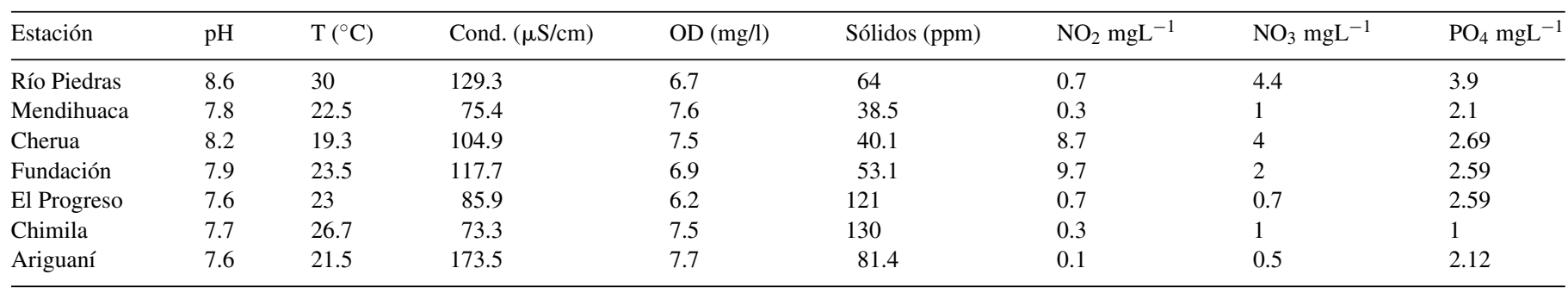


Tabla 3

Abundancia (ind $/ 0.9 \mathrm{~m}^{2}$ ) de familias y géneros de tricópteros encontrados en las diferentes estaciones.

\begin{tabular}{|c|c|c|c|c|c|c|c|c|c|}
\hline \multirow[t]{2}{*}{ Familias } & \multirow[t]{2}{*}{ Géneros } & \multicolumn{8}{|c|}{ Estaciones } \\
\hline & & Río Piedras & Mendihuaca & Cherua & Fundación & El Progreso & Chimila & Ariguaní & Total \\
\hline \multirow[t]{2}{*}{ Hydropsychidae } & Smicridea & 192 & 52 & 63 & 70 & 32 & 9 & 6 & 424 \\
\hline & Leptonema & 1 & 10 & 1 & 14 & 17 & 23 & 81 & 147 \\
\hline \multirow[t]{3}{*}{ Hydroptilidae } & Metrichia & & 5 & 3 & & 4 & & & 12 \\
\hline & Neotrichia & 1 & & & & & & & 1 \\
\hline & Hydroptila & 2 & & & & & & & 2 \\
\hline Philopotamidae & Chimarra & 19 & 10 & & 20 & & 3 & 3 & 55 \\
\hline Hydrobiosidae & Atopsyche & & 3 & 11 & & 1 & & 21 & 36 \\
\hline \multirow[t]{2}{*}{ Glossosomatidae } & Culoptila & & & 10 & & & 3 & 39 & 52 \\
\hline & Protoptila & & 2 & & & & & & 2 \\
\hline Calamoceratidae & Phylloicus & & & 10 & & & & & 10 \\
\hline Helicopsychidae & Helicopsyche & & & 4 & & & & & 4 \\
\hline \multirow[t]{3}{*}{ Leptoceridae } & Atanatolica & 14 & & 49 & 2 & & 8 & 30 & 103 \\
\hline & Neptopsyche & & & & 4 & & & & 4 \\
\hline & Oecetis & 1 & & 4 & 5 & 2 & 2 & & 14 \\
\hline \multirow[t]{2}{*}{ Xiphocentronidae } & Xiphocentron & 2 & & & & & & & 2 \\
\hline & Total & 232 & 82 & 155 & 115 & 56 & 48 & 180 & 868 \\
\hline
\end{tabular}

Se encontraron 868 individuos, asignados a 9 familias y 15 géneros. Hydropsychidae y Leptoceridae fueron las más representativas, con una abundancia del 66 y 14\%, respectivamente. Las familias con menor abundancia relativa fueron Helicopsychidae y Xiphocentronidae, con el 0.4 y $0.2 \%$, respectivamente. A nivel de géneros, Smicridea fue el más representativo con una abundancia del $47 \%$, seguido de Leptonema con el 16\%. Los géneros con menor representación fueron Xiphocentron y Neotrichia con el 0.2 y $0.1 \%$, respectivamente (tabla 3). La estación con mayor riqueza genérica fue Cherua, con 9 géneros, seguida de la estación río Piedras con 8 géneros, mientras que El Progreso fue la estación con menor riqueza, contando solo con 5 géneros. En abundancia, río Piedras fue la estación con mayores valores, contando con 232 ind $/ 0.9 \mathrm{~m}^{2}$; la menor abundancia corresponde a Chimila, con solo $48 \mathrm{ind} / 0.9 \mathrm{~m}^{2}$. Río Piedras posee igualmente los mayores valores de diversidad de Shannon-Weaver (1.423) y equidad de Pielou (0.338) en los índices, mientras que en dominancia, Cherua posee los valores más altos (0.343) (tabla 4).

El análisis de correspondencia canónica explica un porcentaje de varianza del $57.21 \%$ entre los 2 primeros ejes. En el margen izquierdo del gráfico se ubicaron 4 estaciones, en el primer eje Fundación (-1.046), Mendihuaca (-0.442), El Progreso (-0.096), Chimila (-0.016) y río Piedras $(-1.421)$, mientras que a la margen derecha se ubicaron Cherua (1.439)

Tabla 4

Índices de diversidad de las estaciones de muestreo.

\begin{tabular}{llll}
\hline Estación & Dominancia (D) & Shannon $\left(\mathrm{H}^{\prime}\right)$ & Equidad $(\mathrm{J})$ \\
\hline Río Piedras & 0.267 & 1.423 & 0.338 \\
Mendihuaca & 0.143 & 1.250 & 0.148 \\
Cherua & 0.343 & 1.360 & 0.261 \\
Fundación & 0.143 & 1.307 & 0.203 \\
El Progreso & 0.095 & 1.193 & 0.102 \\
Chimila & 0.143 & 1.174 & 0.087 \\
Ariguaní & 0.143 & 1.386 & 0.291 \\
\hline
\end{tabular}

y Ariguaní (0.781); en el margen izquierdo se encuentran 5 variables fisicoquímicas $-\mathrm{pH}$, temperatura, sólidos, fosfatos y nitritos - y 9 géneros de tricópteros - Chimarra, Hydroptila, Leptonema, Neotrichia, Neptopsyche, Oecetis, Protoptila, Smicridea y Xiphocentron-, mientras que en el primer eje del margen derecho se encuentran 3 variables fisicoquímicas - conductividad, OD y nitratos- y 6 géneros -Atanatolica, Atopsyche, Culoptila, Helicopsyche, Metrichia y Phylloicus(fig. 2).

\section{Discusión}

En términos generales, los valores de las variables fisicoquímicas describen las estaciones analizadas como típicos ríos tropicales de alta montaña, con $\mathrm{pH}$ ligeramente básicos, aguas muy oxigenadas y oligotróficas, lo cual permite la autodepuración. La geología de la SNSM se caracteriza por la presencia de rocas ígneas y metamórficas que la proveen con composición alcalina como el granito (Idárraga-García, Posada y Guzmán, 2011), lo que podría explicar los valores ligeramente básicos en todas las estaciones muestreadas. En estudios similares se encontró que el $\mathrm{pH}$, la conductividad, el OD y la temperatura son importantes variables en la distribución de taxones de macroinvertebrados (Carvacho-Aránguiz, 2012; Morelli y Verdi, 2014; Ruiz-García, 2000), y que la mayoría de las especies de Trichoptera son afines a un $\mathrm{pH}$ neutro, mientras que son poco tolerantes a aguas muy alcalinas o muy ácidas (Latorre-Beltrán, Novelo-Gutiérrez y Favila, 2014: Valero et al., 2001). Los valores de conductividad estuvieron ligeramente por encima de los valores registrados para los ríos tropicales de montaña (Roldán, 2012). Estos valores reflejan la naturaleza geoquímica que tiene la SNSM, como se ha mencionado anteriormente. Por otro lado, los valores de nutrientes fueron similares a los registrados en ecosistemas fluviales tropicales conservados (Ortiz-Zayas, Lewis, Saunders, McCutchan y Scatena, 2005). Los valores de fósforo indican que los ríos muestreados son de tipo oligotrófico, sin embargo, los valores de nitritos en Fundación y Cherua fueron 


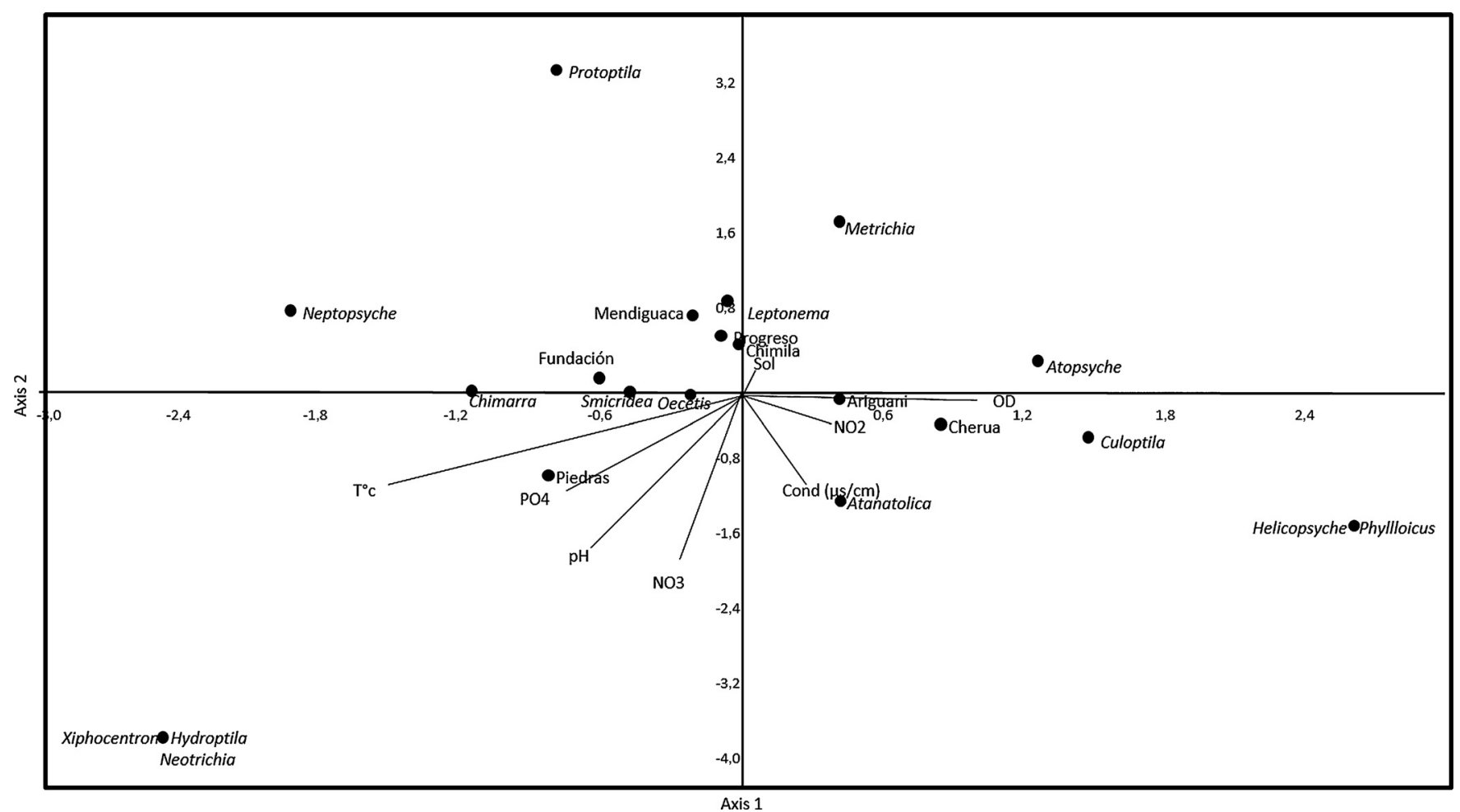

Figura 2. Diagrama del análisis de correspondencia canónica de las relaciones de las estaciones de muestreo con las variables ambientales y los géneros de tricópteros.

altos, debido a las actividades agrícolas, lo que podría afectar a las comunidades de tricópteros a corto y mediano plazos. La carga de nutrientes en los ríos se relaciona directamente con el uso de la tierra y, en especial, con las prácticas agrícolas; históricamente la carga de nutrientes en los afluentes se ha ido incrementando de forma paralela al aumento de las poblaciones humanas en las cuencas, debido a la aplicación de fertilizantes en las tierras de cultivo, con la consiguiente lixiviación a los afluentes (Cooper, 1995).

En este estudio se registraron 9 de las 14 familias de tricópteros presentes en Colombia, misma cifra que registran Muñoz-Quesada, Gutiérrez y Zúñiga (1999) para la SNSM. Sin embargo, cabe aclarar que el estudio de Muñoz-Quesada et al. (1999) fue hecho con adultos. En el caso de los géneros, se registra en este estudio el $68 \%$ de los géneros registrados por Muñoz-Quesada et al. (1999), y el 28\% del total de géneros reportados para Colombia, lo cual constituye un importante aporte al estudio de la diversidad local y regional del orden. Las mayores abundancias de Trichoptera fueron dadas por las familias Hydropsychidae y Leptoceridae, coincidiendo con otros estudios realizados en Colombia (Ballesteros, Zúñiga y Rojas, 1997; Correa, Machado y Roldán, 1981; García, Cantera, Zúñiga y Montoya, 2009; Guevara-Cardona, López-Delgado, Reinoso-Flórez y Villa-Navarro, 2007; Muñoz-Quesada, 2000, 2004; Posada, Roldán y Ramírez, 2000; Vásquez-Ramos, Ramírez-Díaz y Reinoso-Flórez, 2010; Zúñiga, Chará, Giraldo, Chará-Serna y Pedraza, 2013) y el Neotrópico (Angrisano y Sganga, 2009; Springer et al., 2010; Stein, Springer y Kohlmann, 2008). Asimismo, los géneros con mayor abundancia fueron Smicridea y Leptonema; dicha tendencia es similar a lo reportado por varios autores en el Neotrópico, los cuales consideran que las estaciones de menor caudal y orden favorecen mayores abundancias del genero Smicridea, mientras que en la región andina, en rangos altitudinales similares, se observa una tendencia notoria hacia mayores abundancias de Leptonema en cuencas de mayor tamaño y caudal (Ballesteros et al., 1997; Guevara-Cardona et al., 2007; Posada-García y Roldán-Pérez, 2003; Zúñiga, Rojas y Caicedo, 1993), como es el caso de río Piedras y Ariguaní. Una de las posibles explicaciones a la alta abundancia de miembros de la familia Hydropsychidae (Smicridea y Leptonema) en este estudio, puede corresponder a su amplio rango de distribución, ya que esta familia se encuentra asociada a ríos y quebradas con diferentes corrientes y temperaturas (Rincón, 1999), y también por su alta tolerancia a cambios en las condiciones ambientales y su capacidad para construir redes extensas de seda que le permiten fijarse al sustrato y capturar gran cantidad de alimento (López-Delgado et al., 2015). Otra familia con alta abundancia en este estudio fue Leptoceridae; sus especies construyen refugios con material vegetal y mineral como piedras y pequeños trozos de troncos dentro del agua, y presentan diversos comportamientos tróficos, como en las larvas detritívoras o depredadoras, en tanto que algunas se alimentan del perifiton, especialmente de diatomeas que crecen sobre las rocas en zonas de salpicaduras (Muñoz-Quesada, 2004; Roldán, 2012; Wiggins, 1996a).

La composición y riqueza de larvas de tricóptera de los afluentes Cherua y Ariguaní mostraron una variación a nivel espacial, ya que en los tramos altos se registraron los géneros Phylloicus, Helicopsyche, Culoptila, Atopsyche, Atanatolica y Metrichia, que requieren según los resultados de este estudio condiciones de hábitat muy particulares características de zonas altas, como bajas temperaturas, bajos valores de sólidos totales, altos valores 
de oxígeno y pH ligeramente básicos, mientras que en las partes bajas (<300 m snm) están los afluentes Piedra y Fundación, con los géneros Smicridea, Protoptila, Neotrichia entre otros géneros característicos de estas zonas, con altas temperaturas, bajos valores de sólidos totales, altos valores de oxígeno y $\mathrm{pH}$, ligeramente básico. Estos resultados coinciden con lo reportado por Vásquez-Ramos, Guevara y Reinoso-Flórez (2014), quienes estudiaron los factores ambientales asociados con la preferencia de hábitat de larvas de tricópteros en cuencas con bosque seco tropical en el Tolima-Colombia.

A pesar de los valores ligeramente altos de conductividad y de nutrientes para algunos afluentes, los valores de diversidad y abundancia de tricópteros indican el buen estado de los afluentes muestreados en la SNSM. Los resultados de este trabajo indican que los afluentes estudiados constituyen importantes reservorios de diversidad de larvas de tricópteros, a pesar de las actividades agrícolas y urbanas presentadas en algunos. Finalmente, esta investigación corrobora la necesidad de abordar estudios con una mayor intensidad de muestreos y en una mayor escala de tiempo.

\section{Agradecimientos}

A Farid Osorio, Esteffany Barros, Ricardo Martínez, Daniel Cubillos-Alzamora y Anderson Guerrero-Guerrero de la Universidad del Magdalena.

\section{Referencias}

Allan, J. D. y Castillo, M. M. (2007). Stream ecology structure and function of running water (2. ${ }^{\mathrm{a}}$ ed). Netherlands: Springer.

Angrisano, E. B. y Sganga, J. V. (2009). Trichoptera. En E. Domínguez y H. R. Fernández (Eds.), Macroinvertebrados bentónicos sudamericanos. Sistemática y biología (pp. 255-308). San Miguel de Tucumán, Argentina: Fundación Miguel Lillo.

Ballesteros, Y. V., Zúñiga, M. C. y Rojas, A. M. (1997). Distribution and structure of the order Trichoptera in various drainages of the Cauca River basin, Colombia, and their relationships to water quality. En R. W. Holzenthal y O. S. Flint Jr. (Eds.), Proceedings of the 8th International Symposium on Trichoptera (pp. 19-23). Columbus, Ohio: Ohio Biological Survey.

Bispo, P. C., Oliveira, L. G., Crisci-Bispo, V. L. y Sousa, K. G. (2004). Environmental factors influencing distribution and abundance of Trichopteran in Central Brazilian mountain streams. Studies on Neotropical Fauna and Environment, 39, 233-237.

Carvacho-Aránguiz, C. A. (2012). Estudio de las comunidades de macroinvertebrados bentónicos y desarrollo de un índice multimétrico para evaluar el estado ecológico de los ríos de la cuenca del Limari en Chile. Tesis de maestría. Universidad de Barcelona.

Cooper, S. R. (1995). Chesapeake Bay watershed historical land use: impact on water quality and diatom communities. Ecological Applications, 5, 703-723.

Correa, M., Machado, T. y Roldán, G. (1981). Taxonomía y ecología del orden Trichoptera en el Departamento de Antioquia a diferentes pisos altitudinales. Actualidades Biológicas, 10, 35-48.

Diniz-Filho, J. A. F., Oliveira, L. G. y Silva, M. M. (1998). Explaining the beta diversity of aquatic insects in "Cerrado" stream from Central Brazil using multiple Mantel Test. Revista Brasileira de Biologia, 58, 223-231.

Domínguez, E., y Fernández, H. R. (Eds.). (2009). Macroinvertebrados bentónicos sudamericanos. Sistemática y biología. San Miguel de Tucumán, Argentina: Fundación Miguel Lillo.

Dunne, T. y Leopold, L. B. (1978). Water in environmental planning. San Francisco, California: W.H. Freeman and Company.
Fernández, H. R. y Domínguez, E. (2001). Guía para la determinación de los artrópodos bentónicos sudamericanos. San Miguel de Tucumán: Universidad Nacional de Tucumán.

Frissell, C. A., Liss, W. J., Warren, C. E. y Hurley, M. D. (1986). A hierarchical framework for stream habitat classification: viewing streams in a watershed context. Environmental Management, 10, 199-214.

García, J. F., Cantera, J., Zúñiga, M. C. y Montoya, J. (2009). Estructura y diversidad de las comunidades de macroinvertebrados acuáticos en la cuenca baja del río Dagua (Andén Pacífico Vallecaucano-Colombia). Revista de Ciencias de la Universidad del Valle, 13, 27-48.

Guevara, G. (2004). Análisis faunístico del orden Trichoptera en su estado larval en la cuenca del río Coello, Departamento del Tolima. Tesis de maestría. Universidad del Tolima, Ibagué.

Guevara, G., López, E., Reinoso, G., Villa-Navarro, F. (2006). Structure and distribution of the Trichoptera fauna in a Colombian Andean river basin (Prado, Tolima) and their relationship to water quality. Proceedings of the XIIth International Symposium on Trichoptera, Mexico, D.F.

Guevara, G., Reinoso, G., Villa-Navarro, F. (2006). Caddisfly larvae (Insecta: Trichoptera) of the Coello River basin in Tolima (Colombia): spatial and temporal patterns and bioecological aspects. Proceedings of the XIIth International Symposium on Trichoptera, Mexico, D.F.

Guevara-Cardona, G., López-Delgado, E. O., Reinoso-Flórez, G. y VillaNavarro, F. (2007). Structure and distribution of the Trichoptera fauna in a Colombian Andean river basin (Prado, Tolima) and their relationship to water quality. En J. Bueno-Soria, R. Barba-Álvarez, y B. Armitage (Eds.), Proceeding of the XIIth International Symposium on Trichoptera (pp. 129-134). Columbus, Ohio: The Caddis Press.

Hammer, Ø., Harper, D. A. T. y Ryan, P. D. (2001). PAST: Paleontological statistics software package for education and data analysis. Palaentologia Electronica, 4, 1-9.

Huamantinco, A. A. y Nessimian, J. L. (1999). Estrutura e distribuição espacial da comunidade de larvas de Trichoptera (Insecta) em um tributário de primeira ordem do Rio Paquequer, Teresópolis. R.J. Acta Limnologica Brasiliensia, 11, 1-16.

Idárraga-García, J., Posada, B. O. y Guzmán, G. (2011). Geomorfología de la zona costera adyacente al piedemonte occidental de la Sierra Nevada de Santa Marta entre los sectores de Pozos Colorados y Río Córdoba, Caribe colombiano. Boletín de Investigaciones Marinas y Costeras, 40, 41-58.

Latorre-Beltrán, I. T., Novelo-Gutiérrez, R. y Favila, M. E. (2014). Diversidad genérica de Trichoptera (Insecta) en 2 microcuencas del Páramo Rabanal (Cundinamarca-Boyacá, Colombia). Revista de Biología Tropical, 62, 97-110.

López, E. y Reinoso, G. (2008). Variación temporal de la fauna del orden Trichoptera en la cuenca el río Prado. En P. Landázuri (Ed.), Memorias XLIII Congreso Nacional de Ciencias Biológicas. Armenia, Quindío.

López-Delgado, E. O., Vásquez-Ramos, J. M. y Reinoso-Flórez, G. (2015). Listado taxonómico y distribución de los tricópteros inmaduros del departamento del Tolima. Revista de la Academia Colombiana de Ciencias Exactas, Físicas y Naturales, 39, 42-49.

Manjarrés-García, G. y Manjarrés-Pinzón, G. (2004). Contribución al conocimiento hidrobiológico de la parte baja de los ríos de la vertiente noroccidental de la Sierra Nevada de Santa Marta, Colombia. Revista Intropica, 3, 39-50.

Morelli, E. y Verdi, A. (2014). Diversidad de macroinvertebrados acuáticos en cursos de agua dulce con vegetación ribereña nativa de Uruguay. Revista Mexicana de Biodiversidad, 85, 1160-1170.

Muñoz-Quesada, F. (2000). Especies del orden Trichoptera (Insecta) en Colombia. Biota Colombiana, 1, 267-288.

Muñoz-Quesada, F. (2004). El orden Trichoptera (Insecta) en Colombia, II: inmaduros y adultos, consideraciones generales. En F. Fernández, M. G. Andrade, y G. Amat (Eds.), Insectos de Colombia (pp. 319-349). Santafé de Bogotá: Universidad Nacional de Colombia.

Muñoz-Quesada, F., Gutiérrez, L. y Zúñiga, M. C. (1999). Trichoptera from the Sierra Nevada de Santa Marta, Colombia. Bulletin of the North American Benthological Society, 16, 229.

Oliveira, L. G., Bispo, P. C., Crisci, V. L. y Sousa, K. G. (1999). Distribuições de categorias funcionais alimentares de larvas de Trichoptera em uma região serrana do Brasil Central. Acta Limnologica Brasiliensia, 11, 173-183. 
Oliveira, L. G. y Froehlich, C. G. (1996). Natural history of three Hydropsychidae (Trichoptera, Insecta) in a "Cerrado" stream from northeastern São Paulo, Brazil. Revista Brasileira de Zoologia, 13, 755-762.

Ortiz-Zayas, J. R., Lewis, W. M., Jr., Saunders, J. F., III, McCutchan, J. H., Jr. y Scatena, F. N. (2005). Metabolism of a tropical rainforest stream. The North American Benthological Society, 24, 769-783.

Posada, J. A., Roldán, G. y Ramírez, J. J. (2000). Caracterización fisicoquímica y biológica de la calidad de aguas de la cuenca de la Quebrada Piedras Blancas, Antioquia, Colombia. Revista de Biología Tropical, 48, 59-70.

Posada-García, J. A. y Roldán-Pérez, G. (2003). Clave ilustrada y diversidad de las larvas de Trichoptera en el nor-occidente de Colombia. Caldasia, 25, 169-192.

Quintero, A. y Rojas, A. (1987). Aspectos bioecológicos del orden Trichoptera y su relación con la calidad del agua. Revista Colombiana de Entomología, 13, 26-38.

Ramírez, F., Vásquez, J., Reinoso, G. y Guevara, G. (2007). En busca de lo desconocido: una nueva familia en el bentos tolimense (Trichoptera: Xiphocentronidae). En G. Cervantes (Ed.), Memorias del XLII Congreso Nacional de Ciencias Biológicas. . Barranquilla: Departamento de Publicaciones, Universidad Simón Bolívar.

Ramírez, F., Vásquez, J., Reinoso, G. y Guevara, G. (2008). Leptocéridos (Trichoptera) de la cuenca del río Totaré, Tolima-Colombia. En E. Peña, J. Cantera, M. Zúñiga, S. Duque, E. Londoño, L. Aguirre et al. (Eds.), Resúmenes del VIII Seminario Colombiano de Limnología y I Reunión Internacional de Cuencas Bajas y Zonas Estuarinas. Santiago de Cali: Universidad Autónoma de Occidente.

Ramírez, F., Vásquez, J., Reinoso, G. y Guevara, G. (2008b). Los tricópteros y su papel bioindicador: caso del río Totaré. En P. Landázuri (Ed.), Memorias XLIII Congreso Nacional de Ciencias Biológicas. Armenia, Quindío.

Ramírez, A. y Viña, G. (1998). Limnología colombiana. Bogotá, D.C: BP Exploration Colombia y Universidad Jorge Tadeo Lozano.

Reinoso, G. (2001). Estudio bioecológico de los tricópteros del río Alvarado en el trayecto comprendido entre El Salado y Alvarado, Tolima. Ibagué: Universidad del Tolima, Facultad de Ciencias, Departamento de Biología.

Rincón, M. E. (1999). Estudio preliminar de la distribución altitudinal y espacial de los tricópteros en la Cordillera Oriental (Colombia). En G. Amat, M. Andrade, y F. Fernández (Eds.), Insectos de Colombia (pp. 267-284). Bogotá, D.C: II. Academia Colombiana de Ciencias Exactas, Físicas y Naturales.

Roldán, G. P. (2012). Los macroinvertebrados como bioindicadores de la calidad del agua. Bogotá, D.C: Corporación Autónoma Regional de Cundinamarca.

Ruiz-García, A. (2000). Comunidades de tricópteros (Trichoptera) de agua corriente en la provincia de Cádiz. Limnetica, 19, 83-90.

Serna, D. J., Tamaris-Turizo, C. E. y Gutiérrez-Moreno, L. C. (2015). Distribución espacial y temporal de larvas de Trichoptera (Insecta) en el río Manzanares, Sierra Nevada de Santa Marta (Colombia). Revista de Biología Tropical, 63, 465-477.

Spies, M., Froehlich, C. y Kotzian, C. B. (2006). Composition and diversity of Trichoptera (Insecta) larvae communities in the middle section of the Jacuí River and some tributaries, State of Rio Grande do Sul, Brazil. Iheringia, Sér., 96, 389-398.

Springer, M., Ramírez, A. y Hanson, P. (Eds.). (2010). Macroinvertebrados de agua dulce de Costa Rica I. Revista de Biología Tropical, 58.
Stein, H., Springer, M. y Kohlmann, B. (2008). Comparison of two sampling methods for biomonitoring using aquatic macroinvertebrates in the Dos Novillos River, Costa Rica. Ecological Engineering, 34, 267-275.

Valero, L., Durant, P. y Arellano, E. (2001). Trichoptera como indicadora de calidad de agua. Río Albarregas. Mérida, Venezuela. Revista de Ecología Latinoamericana, 8, 11-16.

Vásquez, J. y Ramírez, F. (2008). Aspectos bioecológicos del orden Trichoptera en su estado larval en la cuenca del río Totaré, departamento del Tolima. Tesis. Universidad del Tolima, Facultad de Ciencias Básicas, Departamento de Biología, Ibagué.

Vásquez, J., Ramírez, F., Reinoso, G. y Guevara, G. (2006). Preferencias de sustrato del orden Trichoptera (clase Insecta) en la cuenca del río Combeima, Ibagué-Colombia. En F. Villa, C. Rivera, G. Reinoso, y M. Núñez (Eds.), Resúmenes del VII Seminario Colombiano de Limnología y I Reunión Internacional sobre ríos y humedales neotropicales. Ibagué: Asociación Colombiana de Limnología.

Vásquez, J., Ramírez, F., Reinoso, G. y Guevara, G. (2007). Aspectos bioecológicos del orden Trichoptera en su estado larval en la cuenca del río Totaré Colombia. En G. Cervantes (Ed.), Memorias del XLII Congreso Nacional de Ciencias Biológicas.. Barranquilla: Departamento de Publicaciones, Universidad Simón Bolívar.

Vásquez, J., Ramírez, F., Reinoso, G. y Guevara, G. (2008). Hydroptílidos (Trichoptera) de la cuenca del río Totaré, Tolima-Colombia. En E. Peña, J. Cantera, M. Zúñiga, S. Duque, E. Londoño, L. Aguirre et al. (Eds.), Resúmenes del VIII Seminario Colombiano de Limnología y I Reunión Internacional de Cuencas Bajas y Zonas Estuarinas. Santiago de Cali: Universidad Autónoma de Occidente.

Vásquez, J., Ramírez, F., Reinoso, G. y Guevara, G. (2008b). Estudio de los tricópteros en su estado larval en la cuenca del río Totaré, Tolima, Colombia. En P. Landázuri (Ed.), Memorias XLIII Congreso Nacional de Ciencias Biológicas. Armenia, Quindío.

Vásquez-Ramos, J. M., Guevara, G. y Reinoso-Flórez, G. (2014). Factores ambientales asociados con la preferencia de hábitat de larvas de tricópteros en cuencas con bosque seco tropical (Tolima, Colombia). Revista de Biología Tropical, 62, 21-40.

Vásquez-Ramos, J. M., Ramírez-Díaz, F. y Reinoso-Flórez, G. (2010). Distribución espacial y temporal de los tricópteros inmaduros en la cuenca del río Totaré (Tolima-Colombia). Caldasia, 32, 129-148.

Viloria de la Hoz, J. (2006). Subregiones productivas del Caribe colombiano. Bogotá, D.C: Colección de Economía Regional. Banco de la República.

Wiggins, G. B. (1996a). Larvae of the North American caddisfly genera (Tri-

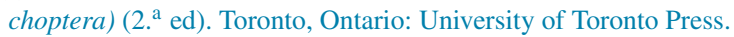

Wiggins, G. B. (1996b). Trichoptera families. En R. W. Merritt y K. W. Cummins (Eds.), An introduction to the aquatic insects of North America (3. ${ }^{\mathrm{a}} \mathrm{ed}, \mathrm{pp}$. 309-349). Dubuque, Iowa: Kendal/Hunt Publishing Company.

Zúñiga, M. C., Chará, J., Giraldo, L. P., Chará-Serna, A. M. y Pedraza, G. X. (2013). Composición de la comunidad de macroinvertebrados acuáticos en pequeñas quebradas de la región andina colombiana, con énfasis en la entomofauna. Dugesiana, 20, 263-277.

Zúñiga, M. C., Rojas, A. M. y Caicedo, G. (1993). Indicadores ambientales de calidad de agua en la cuenca del río Cauca. Ainsa, 13, 17-28. 